\title{
Ética de la peritación estructural de edificios existentes
}

\author{
Ethics on structural surveys for existing buildings
}

\author{
J. Monfort ${ }^{*}$
}

RESUMEN

La amplitud y diversidad de situaciones que se pueden plantear en estos trabajos de peritación, hacen que cualquier norma para regular su contenido omita casos que deben ser resueltos por el perito según su "leal saber y entender". Por la transcendencia y responsabilidad que se puede derivar de ellos, vamos a analizar algunos aspectos vinculados a su repercusión desde el punto de vista legal y profesional.

960-01

Palabras clave: peritación, estructuras de edificación, edificios existentes, seguridad estructural, normas de edificación.

\section{PLANTEAMIENTO}

El carácter genérico del título de este artículo requiere alguna precisión para concretar el contenido que se va a desarrollar.

No se pretende abordar la descripción de posibles patologías, analizando sus causas y soluciones constructivas siguiendo lo que sería un enfoque tecnológico del problema, ni plantear las implicaciones jurídicas que puede tener cualquier informe o dictamen de este tipo. Estos son criterios a tener en cuenta por el perito durante el desarrollo del trabajo, que están suficientemente desarrollados en la bibliografía técnica y en la normativa correspondiente, dejando sólo un corto margen de interpretación por el

\section{SUMMARY}

The breadth and diversity of different situations that may arise in expert's surveys make any rules to regulate its content skip cases that must be solved by the surveyor/expert as its "best knowledge." On the importance and responsibility that can be derived from them, we will analyse some of the aspects related to its implications, in terms of legal and professional point of view.

Keywords: survey, structures of buildings, existing buildings, structural safety, building standards.

carácter subjetivo que tiene cualquier trabajo de este tipo.

Aquí se trata de analizar el problema (personal, conceptual o moral) que se plantea en ocasiones al inicio del trabajo, cuando nos encontramos con un edificio construido en su momento con técnicas y criterios de seguridad que entonces se podían considerar habituales, pero ahora es difícil (o imposible) adecuar (constructiva o económicamente) a la normativa vigente. Esta situación suele presentar cierta lógica constructiva cuando los problemas que se detectan al analizar los distintos elementos estructurales concuerdan con la patología observada (grietas, flechas excesivas, asientos de cimentación, etc.) evidenciando una relación causa-efecto

(*) Universitat Politècnica de València. Valencia, (España) 
que facilita y justifica la decisión a adoptar, del tipo que sea. Pero en otras ocasiones, que no son frecuentes aunque tampoco excesivamente raras, las situaciones anómalas que sólo se detectan mediante catas, ensayos o cálculos de comprobación, es decir son inapreciables a simple vista, no se manifiestan por ningún tipo de patología aparente en un edificio que puede haber estado en uso durante muchos años; esto puede llevar a la contradicción de que una peritación rutinaria en un edificio sin daños evidentes (por adecuación, reforma, cambio de uso, etc.) origina una declaración de ruina o una intervención muy costosa, mientras que otros del mismo período, construidos en la misma zona y previsiblemente con técnicas similares, continúan en uso sin ninguna objeción porque el perito no ha pasado por allí.

El problema deriva, obviamente, de los distintos niveles de exigencia sobre seguridad requeridos en la época de construcción del edificio y en la actualidad, que se enmarca en el ámbito legal y por tanto colectivo; sin embargo, la decisión sobre las secuelas de esta situación es personal del perito y puede dar lugar a distintas interpretaciones de un mismo hecho, tanto por su actitud al redactar el informe, como por la opinión extraída de él por terceras personas tras una lectura meramente literal, que puede entender como resultados científicos lo que se deriva sólo de especulaciones intelectuales.

El análisis de estos aspectos para que la decisión sea lo más objetiva posible es el propósito de este trabajo, y como su origen está en la evolución de las técnicas constructivas y de la normativa vigente, empezaremos revisando estas dos premisas en relación con algunos artículos de la legislación que, por imposibilidad de quedar redactados como normas (reglas que se deben seguir o a que se deben ajustar las conductas, tareas, actividades, etc.) se remiten a principios (cada una de las primeras proposiciones o verdades fundamentales por donde se empiezan a estudiar las ciencias o las artes); la falta de concreción inevitable cuando se pretende aplicar estos criterios en la práctica, tiene que ser interpretada por el perito para enfocar el problema, lo que nos llevará finalmente a hacer algunas consideraciones deontológicas y éticas sobre la actitud o disposición a adoptar ante estas situaciones.

\section{MIRADA POR EL RETROVISOR}

Se ha hecho referencia en el punto anterior a las técnicas y criterios habituales en el momento de la construcción del edificio, que están aceptados de forma explícita por la normativa actual como se recoge el apartado siguiente; muchos de ellos han quedado tan desfasados que parecen de otro siglo (en realidad lo son) a los estudiantes y profesionales recientes en esta actividad, reglada ahora por una normativa detallista y minuciosa con los procedimientos de ensayo y control. A modo de ejemplo, para entender cómo eran estos criterios considerados habituales en su momento, recogemos a continuación algunos párrafos de publicaciones que eran bibliografía de referencia en las Escuelas Técnicas, en un tiempo tan cercano como para que continúen en activo profesionales que se formaron con ellos.

\section{- De "el Bassegoda"(1)}

En el epígrafe Coeficientes de trabajo del terreno, indica:

Al emprender un proyecto hay que cerciorarse de la calidad del terreno, por referencias de obras vecinas $o$, si hace falta, practicando calicatas o exploración con tientaguja o sondeos con barrenas adecuadas. En casos singulares, pueden realizarse ensayos de cargas, siendo ineludibles cuando no se posean datos fidedignos sobre la resistencia del terreno. Al efecto, se carga con lastre un dado de fábrica o una pequeña estampa hasta provocar una presión cosa del doble de la prevista y se mide el asiento consiguiente, el cual no debe exceder de una fracción del tolerable en el edificio, según su destino... Pero la determinación directa de la relación de cargas a asientos es engorrosa y cara; para obviar este inconveniente se aplica la aguja de Stern, en la cual la estampa se substituye por la punta cónica de un pilote de $50 \mathrm{~cm}^{2}$ de sección... Clavada la aguja de acero en el terreno hasta la base del cono, se va cargando paulatinamente hasta alcanzar el doble de la presión unitaria correspondiente a dicha base; el aumento de carga ha de ser de $0,5 \mathrm{~kg}$ cada cinco minutos, en limos y arcillas, y de $1 \mathrm{~kg}$ en el mismo tiempo para los demás terrenos, y por la razón de brazos de palanca los asientos se amplían cinco veces. Finalmente, se rodea la aguja con barro formando un hoyo de $31 \mathrm{~cm}$ de diámetro y 10 $\mathrm{cm}$ de altura y sus caras interiores se mojan durante 12 horas; la aguja no ha de penetrar en la tierra humedecida más de $6 \mathrm{~mm}$, si el asiento tolerable en la construcción es de 30 mm, ni más de $4 \mathrm{~mm}$ si la estructura (bóvedas de puente, altas chimeneas, etc.) no admite más de $10 \mathrm{~mm}$ de asiento. Como coeficiente de trabajo del terreno se considera la mitad de la que transmite por unidad de superficie la base del cono.

Y en el epígrafe Resistencia del hormigón:

La resistencia a compresión puede obtenerse también por ensayos de flexión hasta la rotura sobre prismas copiosamente armados en la 
zona de tracciones; la robustez del refuerzo es causa de que la pieza se rompa por aplastamiento. De la carga de fractura se deduce, por las fórmulas corrientes de flexión simple para el hormigón armado, el valor de la resistencia a la compresión por flexión $B$, que es, en promedio, vez y media la resistencia cúbica. Representando por $W_{\text {e28 }}$ la resistencia cúbica del hormigón húmedo a los 28 días y por $W_{b 28}$ la resistencia cúbica del hormigón, tal como se elabora en la obra, a los 28 días, suelen fijarse los valores siguientes:

a) Hormigón de portland ordinario

$$
W_{\mathrm{e} 28} \geq 200 \mathrm{~kg} / \mathrm{cm}^{2} \quad W_{b 28} \geq 100 \mathrm{~kg} / \mathrm{cm}^{2}
$$

b) Hormigón de supercemento

$$
W_{\mathrm{e} 28} \geq 275 \mathrm{~kg} / \mathrm{cm}^{2} \quad W_{b 28} \geq 130 \mathrm{~kg} / \mathrm{cm}^{2}
$$

Como coeficientes de trabajo se admiten $40 \mathrm{~kg} / \mathrm{cm}^{2}$ en el primer caso y $50 \mathrm{~kg} / \mathrm{cm}^{2}$ en el segundo.

\section{- De "el Benavent" (2)}

El epígrafe Cimientos. Terreno firme. Determinación elemental de la resistencia de un terreno a compresión: fatigas admisibles, dice:

Para conocer aproximadamente la resistencia práctica de un terreno a la compresión, o sea, ..., el número de $\mathrm{kgs}$ por $\mathrm{cm}^{2}$ que aquél es capaz de soportar..., se aplica directamente sobre el mismo terreno, recientemente vaciado y sin apisonar y con intermedio de un soporte de sección conocida (por ejemplo, un tablón de punta), una carga determinada A. Dividiendo la carga A expresada en kgs por la sección $B$ del tablón expresada en $\mathrm{cm}^{2}$ conoceremos aproximadamente la resistencia unitaria del terreno en cuestión: $A / B=500$ ks/100 c $c^{2}$.

A notar que la carga ensayada deberá ser siempre por lo menos una vez y media superior a la carga práctica que se haga soportar al terreno.

En términos generales, puede establecerse que la fatiga admisible a la compresión en un buen terreno corriente para la cimentación no debe apartarse normalmente de 3 ó 4 kgs. por $\mathrm{cm}^{2}$.

\section{- De "el Rodríguez-Avial" (3)}

El epígrafe Valores característicos del acero de construcción, recoge:

En este material, el límite elástico vale, aproximadamente, $\sigma_{E}=20 \mathrm{Kg} / \mathrm{mm}^{2}$, el límite de fluencia, $\sigma_{F}=20$ kilogramos $/ \mathrm{mm}^{2}$, y la carga de rotura varía de 40 a $50 \mathrm{Kg} / \mathrm{mm}^{2}$, valores todos de carácter aproximado.

El valor del límite de fluencia $\sigma_{F}$ es esencial para la determinación de la capacidad resistente del acero de construcción.

De acuerdo con la Instrucción de Estructuras Metálicas redactada por el Instituto de la Construcción "Eduardo Torroja", se tomará como valor del límite de fluencia $\sigma_{F}=2.400$ $\mathrm{Kg} / \mathrm{cm}^{2}$ para barras con espesores no superiores a $20 \mathrm{~mm}$.

Para barras con espesor igual o superior a 30 $\mathrm{mm}$, se tomará $\sigma_{F}=2.300 \mathrm{kilogramos} / \mathrm{cm}^{2}$, pudiéndose interpolar linealmente entre ambos valores para espesores intermedios.

La carga admisible o coeficiente de trabajo generalmente utilizado para lograr la debida seguridad en las estructuras metálicas es de $12 \mathrm{Kg}$ a $14 \mathrm{Kg} / \mathrm{mm}^{2}$ para los esfuerzos de tracción y flexión. En el trabajo de compresión suele valer $12 \mathrm{Kg} / \mathrm{mm}^{2}$.

- De "el Moral"( 4)

En el epígrafe Coeficientes de seguridad y tensiones admisibles:

Para fijar los coeficientes de seguridad, deberá comenzarse por estudiar las características y circunstancias especiales de la obra. Así, pues, no deberán adoptarse iguales coeficientes de seguridad para una obra provisional que para una definitiva, ni para una pequeña obra cuya ruina no pueda ocasionar grandes perjuicios, o para otra cuya destrucción pueda originar una verdadera catástrofe, como la rotura de una gran presa, ni tampoco para una obra que haya de ejecutarse sin la debida inspección y casi sin la presencia del técnico, o en aquella en que se ejerza una inspección rigurosa y hasta se disponga de un pequeño laboratorio que permita ir comprobando en cada momento las condiciones de los materiales que se emplean; ni en una obra proyectada de prisa y corriendo en que sólo se han considerado los esfuerzos más importantes y otra proyectada con todo detalle y cuidado, en la cual se hayan tenido en cuenta escrupulosamente todas las causas externas e internas que pueden influir en ella.

Por estas razones recomendamos que antes de empezar un proyecto se medite detenidamente sobre esta cuestión, que, por otra parte, puede tener mayor importancia económica que la buena disposición del proyecto y la exactitud de los cálculos, puesto que de considerar un hormigón trabajando a $40 \mathrm{~kg} /$ $\mathrm{cm}^{2}$ a considerarlo a $65 \mathrm{~kg} / \mathrm{cm}^{2}$, y aún más, pueden existir diferencias en coste del orden de un 100 por 100. 
Y en el epígrafe Armaduras:

Casi siempre las barras que forman la armadura principal del pilar se disponen a lo largo de dos caras opuestas del mismo; es decir, sobre dos ejes perpendiculares al de máxima flexión, y decimos en dos caras, aun cuando en realidad sólo serían necesarias en la extendida, porque es difícil encontrar pilares en los que por efecto de las distintas hipótesis de carga no pueda admitirse una inversión completa de los efectos de flexión.

No obstante, en aquellos casos en que las flexiones se hallen perfectamente determinadas, y correspondan las tracciones en la mitad superior del pilar a una cara y en la inferior a la opuesta, no hay inconveniente ninguno en disponer un solo plano de armadura, que pase alternativamente de una a otra de las caras del pilar, buscando las zonas de tracciones máximas.

\section{NORMATIVA ACTUAL}

Aunque estamos hablando de la peritación, un estudio que se formaliza en un informe o dictamen, de ella puede derivarse la ejecución de obras de adecuación estructural, por lo que es lógico tomar como referencia la normativa que deben cumplir estas obras en caso de ser necesarias.

El Código Técnico de la Edificación, en el Capítulo 1 Disposiciones generales, Artículo 2 Ámbito de aplicación, establece:

3- ...el CTE se aplicará a las obras de ampliación, modificación, reforma o rehabilitación que se realicen en edificios existentes, siempre y cuando dichas obras sean compatibles con la naturaleza de la intervención y, en su caso, con el grado de protección que puedan tener los edificios afectados.

$$
\ldots
$$

y en el punto 4 del mismo artículo puntualiza estos aspectos,

4- A estos efectos, se entenderá por obras de rehabilitación aquéllas que tengan por objeto actuaciones tendentes a lograr alguno de los siguientes resultados:

a) La adecuación estructural, considerando como tal las obras que proporcionen al edificio condiciones de seguridad constructiva, de forma que quede garantizada su estabilidad y resistencia mecánica

por lo que, salvo en edificios con algún grado de protección, el CTE es aplicable en la actualidad a los trabajos de peritación estructural. No obstante, como la mayor parte de su extenso contenido está dedicado a las prestaciones exigibles a la obra nueva y cuando se trata de intervenciones en edificios existentes se producen particularidades que afectan tanto a estas prestaciones como al tipo de trabajos que se pueden realizar, en el Documento Básico DB SE Seguridad Estructural incluye el Anejo D Evaluación estructural de edificios existentes, donde puntualiza estos aspectos puesto que, como indica su artículo D.1.1 Ámbito de aplicación, en la evaluación estructural de edificios existentes puede existir un mayor grado de diferenciación de la seguridad que para el dimensionado estructural de edificios de nueva construcción, debido a consideraciones de tipo económico, social o medioambiental. En otro punto del mismo artículo establece:

2- Los criterios generales establecidos en este Anejo son aplicables para la evaluación estructural de cualquier tipo de edificio existente, si se cumple alguna de las siguientes condiciones:

a) se ha concebido, dimensionado y construido de acuerdo con las reglas en vigor en el momento de su realización;

b) se ha construido de acuerdo con la buena práctica, la experiencia histórica y la práctica profesional aceptada

en edificios con cierta antigüedad no se cumple al apartado a) simplemente porque no existían reglas en vigor, pero se suele cumplir el b) por lo que seguiremos esta línea.

Este mismo Anejo D, en el artículo D.1.2 Consideraciones previas, indica:

1- No es necesaria la utilización directa de las normas y reglas establecidas en este CTE en la evaluación estructural de edificios existentes, construidos en base a reglas anteriores a las actuales para los edificios de nueva construcción, por los siguientes motivos: ...

d) las normas actuales suelen estar basadas en exigencias diferentes y generalmente más estrictas que las vigentes en el momento en que se proyectó el edificio, por lo cual, muchos edificios existentes se clasificarían como no fiables si se evaluaran según las normas actuales;

e) se puede considerar, en muchos casos, un período de servicio reducido, lo que se traduce también en una reducción de las exigencias;

f) se pueden emplear modelos de análisis más afinados (a través de inspecciones, ensayos, mediciones in situ o consideraciones teóricas), lo que puede aportar beneficios adicionales

en consecuencia, a pesar de la indicación del artículo inicial sobre que el CTE se aplicará a las obras de ampliación, modificación, reforma o rehabilitación que se realicen en 
edificios existentes, aquí matiza que no es necesaria la utilización directa de las normas y reglas establecidas en este CTE en la evaluación estructural de edificios existentes, o sea, que se aplicará pero "interpretándolo" (esta matización se concreta con la diferenciación entre criterios de evaluación y de refuerzo, recogidos al final de este epígrafe al referirse a las medidas constructivas); no obstante, deja claro que muchos edificios existentes se clasificarían como no fiables si se evaluaran según las normas actuales, y no es ese el objetivo, por lo que la interpretación anterior debe buscar una solución razonable a este problema. La definición de ese criterio queda pendiente por lo visto hasta ahora.

En el artículo D.2 Criterios básicos para la evaluación, admite dos procedimientos para verificar la capacidad portante: cuantitativos y cualitativos. Especifica con mayor detalle y da prioridad implícitamente a los cuantitativos, para los que reitera en dos puntos distintos un aspecto importante:

- en D.2.3 Especificación de los objetivos

1- Antes del inicio de la evaluación deben establecerse claramente los objetivos de la misma, en términos de las prestaciones futuras del edificio, definidas éstas a partir de las siguientes exigencias:

a) el nivel de seguridad en relación con la resistencia y la estabilidad estructural.

- en D.5.2.2 Evaluación detallada

...

2- Los coeficientes parciales particularizados se calibrarán para que sean consistentes con el nivel requerido de seguridad estructural. Normalmente serán menos conservadores que los coeficientes correspondientes incluidos en los documentos básicos correspondientes para el dimensionado de edificios de nueva construcción.

Es decir, admite explícitamente coeficientes de seguridad inferiores a los establecidos para obra nueva, pero no cuantifica su valor.

Estos procedimientos cuantitativos presentan un problema importante en un caso muy frecuente, como es el de los pórticos de hormigón armado con nudos rígidos: cuando no se dispone de información fiable sobre la cuantía y posición de las armaduras, y sus longitudes de anclaje, la rigidez de los nudos no está garantizada y este factor tiene gran influencia en los resultados que se obtienen del cálculo, por lo que éstos tampoco son demasiado fiables. Debido a ello, también se pueden utilizar los procedimientos cualitativos para la evaluación estructural que recoge este Anejo D; así, en el artículo D.2.1 Procedimiento, indica:
2- En edificios en los que no resulte posible o sea poco fiable una verificación cuantitativa, o cuando el edificio haya demostrado un comportamiento satisfactorio en el pasado, podrá realizarse una evaluación cualitativa de la capacidad portante y de la aptitud de servicio de acuerdo con los criterios enumerados de D. 6

y en este apartado D.6 Evaluación cualitativa, concreta:

\section{- D.6.1 Capacidad portante}

1- Puede suponerse que un edificio que haya sido dimensionado y construido de acuerdo con las reglas de normas antiguas, tendrá una capacidad portante adecuada, si se cumplen las siguientes condiciones:

a) el edificio se ha utilizado durante un período de tiempo suficientemente largo sin que se hayan producido daños o anomalías (desplazamientos, deformaciones, fisuras, corrosión, etc.);

b) una inspección detallada no revele ningún indicio de daños o deterioro;

c) la revisión del sistema constructivo permita asegurar una transmisión adecuada de las fuerzas, especialmente a través de los detalles críticos;

d) teniendo en cuenta el deterioro previsible así como el programa de mantenimiento previsto se puede anticipar una durabilidad adecuada;

e) durante un período de tiempo suficientemente largo no se han producido cambios que pudieran haber incrementado las acciones sobre el edificio o haber afectado su durabilidad;

f) durante el período de servicio restante no se prevean cambios que pudieran incrementar las acciones sobre el edificio o afectar su durabilidad de manera significativa.

2- Una evaluación cualitativa de la capacidad portante de un edificio existente puede ser insuficiente para situaciones de dimensionado extraordinarias.

3- El comportamiento de un edificio cuya capacidad portante haya sido evaluada cualitativamente se controlará periódicamente durante el período de servicio restante. Para ello se emplearán los medios que se estimen necesarios, dependiendo de las características de la estructura, así como de las acciones e influencias que actúen sobre ella y de su estado.

- En D.6.2 Aptitud al servicio, impone para esta situación algunas condiciones que son reiterativas con las que se acaban de citar (apartados a), b), d) y f) del punto 1) 
Con referencia a estas condiciones, la 1-c) relativa a la transmisión adecuada de fuerzas a través de los detalles críticos sigue teniendo la dificultad de verificación que se ha indicado antes para algunos casos concretos y frecuentes, como son los nudos rígidos en estructuras de hormigón armado; las relativas a problemas de durabilidad, si existen, se pueden mejorar con técnicas constructivas habituales hoy en día, y el control periódico requerido se puede especificar en el programa de mantenimiento posterior del edificio.

Sobre la exclusión que prácticamente hace de la validez de estos procedimientos cualitativos para comprobar situaciones extraordinarias, se debe tener en cuenta que las de este tipo a considerar en los edificios son dos: fuego y sismo. La protección contra el fuego se puede conseguir con los tratamientos habituales que se aplican actualmente para alcanzar las prestaciones que exige el Documento Básico DB SI Seguridad en caso de Incendio (con la única dificultad añadida que supone aplicarlos en una construcción existente); sobre el sismo, la vigente Norma de Construcción Sismorresistente NCSE-02, en el artículo 1.2.1 Ámbito de aplicación, establece:

Esta Norma es de aplicación al proyecto, construcción y conservación de edificaciones de nueva planta. En los casos de reforma o rehabilitación se tendrá en cuenta esta Norma, a fin de que los niveles de seguridad de los elementos afectados sean superiores a los que poseían en su concepción original. Las obras de rehabilitación o reforma que impliquen modificaciones substanciales de la estructura (por ejemplo: vaciado de interior dejando sólo la fachada), son asimilables a todos los efectos a las de construcción de nueva planta.

es decir, con carácter general excluye de su ámbito de aplicación los casos de reforma o rehabilitación, o lo que es equivalente, considera suficiente cualquier tipo de mejora que aumente el nivel de seguridad frente a este tipo de acción; sólo debe aplicarse en casos como el ejemplo citado, vaciado de interior dejando sólo la fachada (por protección patrimonial o urbanística, normalmente), que supone la renovación completa de la estructura y el único problema a tener en cuenta es su conexión con la fachada existente.

Finalmente, el Anejo D del CTE recoge, independientemente de que la evaluación se haya realizado por un procedimiento cuantitativo o cualitativo, las medidas a adoptar para asegurar, restablecer o mantener la seguridad estructural de un edificio que, según el caso, podrán ser de tres tipos: de aseguramiento estructural, técnico-administrativas o constructivas; con respecto a éstas últimas es importante señalar que el artículo D.8.3 Medidas constructivas, indica: ...

2- Los elementos de refuerzo de una estructura se dimensionarán según las especificaciones para el dimensionado estructural de edificios de nueva construcción. Alternativamente, las verificaciones relativas a los elementos de refuerzo se podrán basar en una aplicación directa de los métodos de análisis de seguridad.

es decir, si como consecuencia de la peritación es necesario realizar algún refuerzo en la estructura, estos elementos sí que deben proyectarse siguiendo las especificaciones del CTE para obra nueva. Con lo cual diferencia claramente entre los criterios de evaluación aplicables para justificar la seguridad de una estructura existente, o parte de ella, y los que se deben tener en cuenta para proyectar los elementos de refuerzo que eventualmente puedan ser necesarios.

Como resumen de este conjunto de disposiciones normativas, muchos de los casos que se suelen plantear en peritaciones están incluidos en las situaciones descritas y no plantean problemas de interpretación; pero otros, numerosos por la antigüedad de bastantes edificios en uso, requieren criterios para evaluar aspectos que el texto legal no concreta, como algunos ya señalados:

- los valores del coeficiente de seguridad, en caso de comprobación cuantitativa

- la estimación de la rigidez de los nudos en estructuras de hormigón armado para una comprobación cuantitativa

- la transmisión adecuada de fuerzas a través de los detalles críticos, en una comprobación cualitativa

y es en este ámbito donde el perito debe adoptar decisiones trascendentes.

\section{DEONTOLOGÍA}

Buscando algún fundamento que permita adoptar estas decisiones a partir de alguna referencia para que no queden en una cuestión subjetiva, se puede recurrir a la Deontología que, en general aunque con distintos tratamientos, regula todas las titulaciones que facultan para ejercer la actividad de perito en este campo.

La Deontología es la rama de la Ética que estudia los fundamentos del deber y las normas morales, por lo que también se conoce como "teoría del deber" o "ciencia de lo debido"; sólo considera los actos humanos voluntarios 
y libres, mediante una reflexión teórica entre la moral y el derecho, para ordenar el ámbito donde se desarrollan.

Para concretar su campo de actuación, pasando de las ideas generales a normas descriptivas o prescriptivas, la deontología profesional estudia los derechos y deberes relativos al ejercicio de una profesión, en especial aquéllos que no están delimitados jurídicamente, y los explicita mediante códigos aplicables sólo a los miembros del colectivo; en nuestro caso se formalizan a través de los Colegios profesionales, Corporaciones de derecho público, amparadas por la Ley y reconocidas por el Estado, con personalidad jurídica propia y plena capacidad para el cumplimiento de sus fines ... entre los que se encuentra la ordenación del ejercicio de las profesiones (5).

En la práctica los códigos deontológicos de los Colegios que regulan las actividades de carácter técnico, se ocupan de los asuntos administrativos, de régimen colegial interno y competenciales, y no de temas tan generales como el planteado en este caso, que no crea conflictos entre el colectivo.

Como la Deontología enfocada hacia su concreción práctica como es el ámbito profesional, no da respuesta para los criterios objetivos de evaluación que vamos buscando, seguiremos el camino inverso generalizando su estudio en el campo más amplio de la Ética.

\section{5. ÉTICA}

Es la parte de la filosofía que trata de la moral y de las obligaciones del hombre; los conceptos de ética y moral aparecen siempre vinculados (etimológicamente ambos se definen como "ciencia de las costumbres", con raíz latina uno y griega el otro) aunque presentan diferencias: la moral nace en el seno de una sociedad y ejerce influencia en la conducta de cada uno de sus integrantes, mientras que la ética es un valor captado y apreciado internamente por la reflexión consciente y voluntaria de un sujeto, que puede coincidir o no con la moral recibida.

Cuando se formaliza hacia situaciones concretas como puede ser la ética profesional, no debe confundirse con la Deontología, recogida en normas y códigos como se ha indicado, mientras que la ética sólo está relacionada con lo que piensa el propio individuo constituyendo una conciencia profesional, que sólo se puede plantear en términos de principios (6) mucho más genéricos que las normas:

- principio de beneficencia: comprobar si se logra y cómo se logra materializar los bienes y proporcionar los servicios
- principio de autonomía: respetar la dignidad, autonomía y derechos de las personas a las que va destinada la actuación profesional

- principio de justicia: jerarquizar los diversos condicionantes que afectan al ámbito del trabajo y administrar razonablemente los recursos limitados

- principio de no maleficencia: evitar cualquier daño o perjuicio a toda persona afectada por la actuación

como consecuencia de todo ello, la conciencia profesional es reflexiva (consciente de sí misma), intransferible e individual como la conciencia humana (ética), pero le añade, además, la responsabilidad que cada persona tiene.

Obviamente, en este ámbito general se puede abordar cualquier problema, como los criterios de evaluación estructural a los que hemos hecho referencia antes; pero buscábamos una solución objetiva para situaciones que no están contempladas en ninguna norma y aquí se enfrenta el problema con la conciencia individual, es decir subjetivamente.

No es la solución deseada, pero era previsible que para los problemas singulares no previstos en ningún reglamento los criterios de evaluación hayan de ser personales.

\section{CONCLUSIÓN (Y COROLARIO)}

Este trabajo es una reflexión para llegar a un punto previsto desde el principio, que ha permitido analizar progresivamente los pasos a través de distintas disciplinas, que se van introduciendo a medida que los criterios previos resultan insuficientes; esto permite ubicar los diferentes tipos de problemas en su marco normativo, cuando está previsto, y en consecuencia tratar subjetivamente sólo los casos, o la parte de ellos, que sea estrictamente necesario por carecer de cualquier referencia objetiva.

Por tanto sucede como en cualquier campo de la actividad humana: la conciencia colectiva, adquirida por procedimientos no demasiado precisos, es consciente de que unas cosas están bien, otras mal, y finalmente hay algunas sobre las que no existe un criterio extendido y cada uno las debe afrontar con un enfoque personal. Aplicado al tema que nos ocupa, la profesionalidad y el bien hacer del perito consiste en identificar correctamente cada tipo de problema, como reglado o no reglado, aplicando la norma correspondiente a los primeros y evaluando razonablemente desde su punto de vista los criterios a considerar, sólo cuando sea necesario, en los segundos. 
Lo que da relevancia a este ámbito de actuación es la responsabilidad y trascendencia de las decisiones que se adoptan:

- responsabilidad, del perito, por las implicaciones legales de un posible fallo estructural consecuencia, en algunos casos, de una decisión subjetiva no amparada por ninguna regla o norma

- trascendencia, para los usuarios del edificio, por la posible declaración de ruina o intervención muy costosa económicamente debida al informe emitido

con la dificultad añadida de tener que adoptar la decisión enfrentando dos alternativas tan radicales cuando, en ocasiones, los daños aparentes no concuerdan con los datos que se obtienen del estudio pormenorizado de los materiales y elementos.

Esta aparente elucubración para buscar algún caso que se aparte de la regla general y, por ello, constituya un problema, no se puede considerar como un hecho aislado carente de relevancia; la construcción de las estructuras de edificación ha cambiado de nivel y lo que antes se consideraba objeto de un oficio ahora es tratado como una ciencia, y además esto se ha producido en un período de tiempo lo bastante corto como para que muchos edificios construidos con el criterio anterior sigan en uso actualmente, y con frecuencia son el objeto de las peritaciones a que me estoy refiriendo.

Se produce entonces la contradicción, o al menos una duda razonable, por el hecho de que los métodos del análisis actual que se aplican en la peritación no tienen nada que ver con los procedimientos de proyecto y ejecución empleados en su construcción y esto hace que, muchas veces, el contenido del informe que resulta no sea más que una opinión, de un experto pero no por ello deja de ser cuestionable (al menos por otro experto), que con los correspondientes visados colegiales, registros de entrada en organismos oficiales o alguna otra cosa parecida, se formaliza en un documento que aparenta una infalibilidad de la que en realidad carece. Y esta creo que es la verdadera conclusión: el carácter opinable de muchos informes periciales, avalados por ensayos y cálculos aceptados en "este" tiempo pero de validez relativa cuando se aplican a edificios de "otro" tiempo.

Aunque la conclusión final era evidente desde el principio, llegar a ella a través de sus argumentos refuerza su consistencia. Eso he pretendido.

\section{BIBLIOGRAFÍA}

(1) Schindler, R. versión del alemán y adiciones B. Bassegoda: Tratado moderno de construcción de edificios. p. 8-9 y 334, José Montesó Editor, Barcelona, séptima edición, 1963.

(2) Benavent, P.: Como debo construir. p. 33, Bosch Casa Editorial, Barcelona, sexta edición, 1963.

(3) Rodríguez-Avial, F.: Construcciones metálicas. p. 13, Sección de Publicaciones de la E.T.S. de Ingenieros Industriales, Madrid, sexta edición, 1968.

(4) Moral, F.: Hormigón armado. p. 58-59 y 338, Editorial Dossat S.A., Madrid, sexta edición, 1962.

(5) Ley 2/1974, de 13 de febrero, sobre Colegios Profesionales. Artículo 1

(6) Informe Belmont: Principios éticos y pautas para la protección de los seres humanos en la investigación. Departamento de Salud, Educación y Bienestar USA, 1979, adoptado como Regla Común por 14 Departamentos Federales en 1991 